\title{
Ferromagnetism in the Hubbard model with long-range and correlated hopping
}

\author{
Pavol Farkašovský \\ Institute of Experimental Physics, Slovak Academy of Sciences \\ Watsonova 47, 04353 Košice, Slovakia
}

\begin{abstract}
The extrapolation of small-cluster exact-diagonalization calculations is used to examine ferromagnetism in the one-dimensional Hubbard model with long-range and correlated hopping. It is found that the correlated hopping term stabilizes the ferromagnetic state for a wide range of electron interactions $U$ and electron concentrations $n$. The critical value of the interaction strength $U_{c}$ above which the ferromagnetic state becomes stable is calculated numerically and the ground-state phase diagram of the model is presented for physically the most interesting cases.
\end{abstract}


The Hubbard model has become, since its inception [1] in 1963, one of the most popular examples of a system of interacting electrons with short-range interactions. It has been used in the literature to study a great variety of many-body effects in metals, of which ferromagnetism, metal-insulator transitions, charge-density waves and superconductivity are the most common examples. Of all these cooperative phenomena the problem of ferromagnetism in the Hubbard model has the longest history. Although the model was originally introduced to describe the band ferromagnetism of transition metals, it soon turned out that the single-band Hubbard model is not the canonical model for ferromagnetism. In fact the existence of saturated ferromagnetism has been proven rigorously only for very special limits. The first well-known example is the Nagaoka ferromagnetism that comes from the Hubbard model in the limit of infinite repulsion and one hole in a half-filled band [2]. Another example where saturated ferromagnetism has been shown to exist is the case of the one-dimensional Hubbard model with nearest and nextnearest-neighbor hopping at low electron densities [3]. Moreover, several examples of the fully polarized ground state have been found on special lattices (special conduction bands) as are the fcc-type lattices [4, the bipartite lattices with sublattices containing a different number of sites [5], the lattices with unconstrained hopping of electrons [6] and the flat bands [7]. This indicates that the lattice structure and the kinetic energy of electrons, i.e., the type of hopping play an important role in stabilizing the ferromagnetic state.

In our previous paper [8] we have shown that if the electron hopping is described by a more realistic model (than the nearest-neighbor hopping) then ferromagnetism comes from the Hubbard model naturally for a wide range of the model parameters. No extra interactions terms should be included. In particular, we have found that the long-range hopping with power decaying hopping amplitudes $t_{i j}$ given by 9 ] 


$$
t_{i j}(q)=\left\{\begin{array}{cc}
0, & i=j, \\
-q^{|i-j|} / q, & i \neq j
\end{array}\right.
$$

gives rise to ferromagnetism for electron densities above half-filling. As soon as $q$ that controls the effective range of the hopping $(0 \leq q \leq 1)$ is different from zero the ferromagnetic state is stabilized for all Coulomb interactions $U$ greater than some critical interaction strength $U_{c}(q)$ that value is dramatically reduced with increasing $q$. From this point of view one of main reasons why the ferromagnetic state absent in the ordinary Hubbard model with nearest-neighbor hopping $(q=0)$ is that the description of the electron hopping was too simplified.

Here we further generalize this model by introducing the correlated hopping term, in which the $\sigma$-electron hopping amplitudes between lattice sites $i$ and $j$ depend explicitly on $n_{i-\sigma}$ and $n_{j-\sigma}$ occupancy, i.e.

$$
t_{i j}^{\sigma}=t_{i j}\left[1+t^{\prime}\left(n_{i-\sigma}+n_{j-\sigma}\right)\right]
$$

The importance of the correlated hopping term on the ground-state properties of the Hubbard model has been already mentioned by Hubbard [1]. Later Hirsch [10] pointed out that this term may be relevant in explanation of superconducting properties of strongly correlated electrons. Here we examine effects of this term on the stability of the fully polarized ferromagnetic state. The same subject has been studied recently by Amadon and Hirch [11], as well as by Kollar and Vollhardt [12, however they considered hopping only between the nearest-neighbor sites.

The selection of hopping matrix elements in the form given by Eq. 1 and Eq. 2 has several advantages. It represents a much more realistic type of electron hopping on a lattice (in comparison to nearest-neighbor hopping), and it allows us to change continuously the type of hopping and thus immediately study the effect of long- 
range and correlated hopping on the ground state properties of the Hubbard model.

The Hamiltonian of the single-band Hubbard model in which the effects of long-range and correlated hopping are incorporated is given by

$$
H=\sum_{i j \sigma} t_{i j}^{\sigma} c_{i \sigma}^{+} c_{j \sigma}+U \sum_{i} n_{i \uparrow} n_{i \downarrow}
$$

where $c_{i \sigma}^{+}$and $c_{i \sigma}$ are the creation and annihilation operators for an electron of spin $\sigma$ at site $i, n_{i \sigma}$ is the corresponding number operator $\left(N_{\sigma}=\sum_{i} n_{i \sigma}\right)$ and $U$ is the on-site Coulomb interaction constant.

To examine possibilities for existence of ferromagnetism in this model the ground states are determined by exact diagonalizations for a wide range of model parameters $\left(q, t^{\prime}, U, N=\sum_{\sigma} N_{\sigma}\right)$. Typical examples are then chosen from a large number of available results to represent the most interesting cases. The results obtained are presented in the form of phase diagrams in the $U-q$ plane. To determine the phase diagram in the $U-q$ plane (corresponding to some $t^{\prime}$ and $N$ ) the ground state energy of the model is calculated point by point as functions of $q$ and $U$. Of course, such a procedure demands in practice a considerable amount of CPU time, which imposes severe restrictions on the size of clusters $(L)$ that can be studied with this method $(L \sim 16)$. Fortunately, we have found that the ground-state energy of the model depends on $L$ only very weakly (for a wide range of the model parameters) and thus already such small clusters can describe satisfactorily the ground state properties of the model.

The results of our small-cluster exact-diagonalization calculations obtained on finite clusters up to $L=16$ sites are summarized in Fig. 1 and Fig. 2. There is shown the critical interaction strength $U_{c}$, above which the ground state is ferromagnetic, as a function of $q$ for selected values of $n=N / L$ and $t^{\prime}\left(n=3 / 2,7 / 4 ; t^{\prime}=\right.$ $0,0.2,0.4)$. To reveal the finite-size effects on the stability of ferromagnetic do- 
mains, the behavior of the critical interaction strength $U_{c}(q)$ has been calculated on several finite clusters at each electron filling. It is seen that finite-size effects on $U_{c}$ are small and thus these results can be satisfactorily extrapolated to the thermodynamic limit $L \rightarrow \infty$. Our results clearly demonstrate that the ferromagnetic state is strongly influenced by correlated hopping $\left(t^{\prime}\right)$ and generally it is stabilized with increasing $t^{\prime}$. The effect is especially strong for intermediate and strong values of $q$. Even, there exists some critical value of $q$ above which the ground state is ferromagnetic for all nonzero $U$. With increasing $t^{\prime}$ this critical value shifts to lower values of $q$ (that represent a much more realistic type of electron hopping) and the ferromagnetic domain correspondingly increases. Performing exhaustive numerical studies of the model for a wide range of electron concentrations (on different lattice clusters) we have found that the model exhibits the same behavior for all electron concentrations above half-filling [13, and that with increasing concentration this effect becomes more pronounced (see Fig. 1 and Fig. 2). These results clearly show that ferromagnetism comes naturally from the Hubbard model with long-range and correlated hopping for a wide range of model parameters without any other assumptions. This opens a new route towards the understanding of ferromagnetism in the Hubbard model.

In summary, the extrapolation of small-cluster exact-diagonalization calculations was used to examine ferromagnetism in the one-dimensional Hubbard model with long-range and correlated hopping. It was found that the correlated hopping term stabilizes the ferromagnetic state for a wide range of electron interactions $U$ and electron concentrations $n$. The critical value of the interaction strength $U_{c}$ above which the ferromagnetic state becomes stable was calculated numerically and the ground-state phase diagram of the model is presented for physically the most interesting cases. 
This work was supported by the Slovak Grant Agency VEGA under Grant No. 2/7021/20 and the Science and Technology Assistance Agency under Grant APVT-51-021602. Numerical results were obtained using computational resources of the Computing Centre of the Slovak Academy of Sciences. 


\section{References}

[1] J. Hubbard, Proc. R. Soc. London A 276, 238 (1963).

[2] Y. Nagaoka, Phys. Rev. 147, 392 (1966).

[3] E. Múller-Hartmann, J. Low. Temp. Phys.99, 342 (1995).

[4] M. Ulmke, Eur. Phys. J. B 1, 301 (1998).

[5] E.H. Lieb, Phys. Rev. Lett. 62, 1201 (1989).

[6] P. Pieri, Mod. Phys. Lett. B10, 1277 (1996); M. Salerno, Z. Phys. B 99, 469 (1996); ibid. B 101, 619 (1996).

[7] A. Mielke and H. Tasaki, Commun. Math. Phys. 158, 341 (1993).

[8] P. Farkašovský, Phys. Rev. B 66, 012404 (2002).

[9] P. Farkašovský, J. Phys. Condens. Matter 7, 3001 (1995); ibid. 7, 9775 (1995);

Phys. Rev. B 57, 14722 (1998); Int. J. Mod. Phys. B 12, 803 (1998).

[10] J.E. Hirsch, Physica C 158, 236 (1989).

[11] J.C. Amadon and J.E. Hirsch, Phys. Rev. B 54, 6364 (1996).

[12] M. Kollar and D. Vollhardt, Phys. Rev. B 63, 045107 (2001).

[13] $n \leq 1$ and $t^{\prime}<0$ does not stabilize the ferromagnetic state. 


\section{Figure Captions}

Fig. 1. The critical interaction strength $U_{c}$ as a function of $q$ calculated for different $t^{\prime}$ and $L$ at $n=3 / 2$. Curves from up to down correspond to: $t^{\prime}=0,0.2$ and 0.4 .

Fig. 2. The critical interaction strength $U_{c}$ as a function of $q$ calculated for different $t^{\prime}$ and $L$ at $n=7 / 4$. Curves from up to down correspond to: $t^{\prime}=0,0.2$ and 0.4 


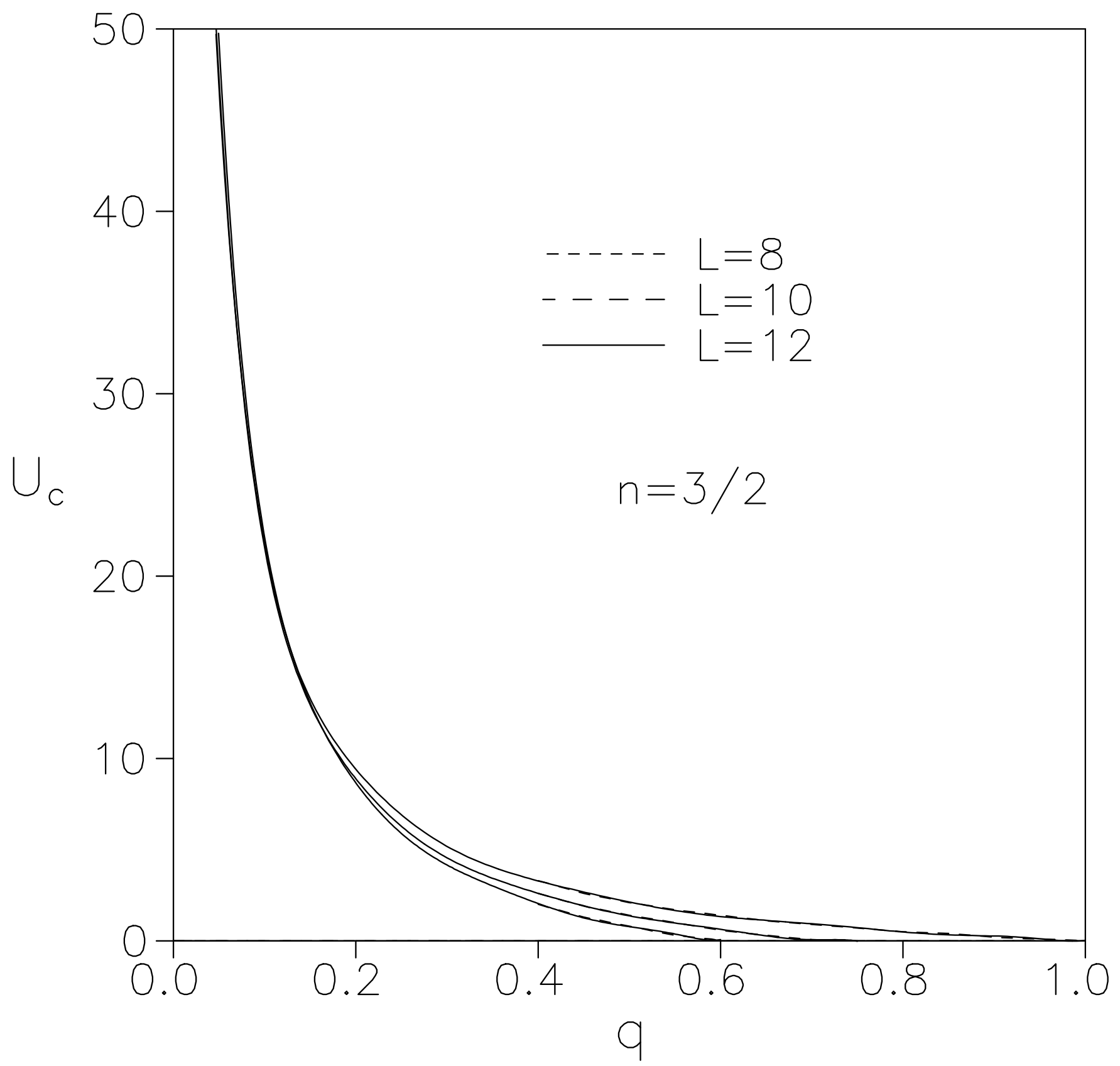

Figure 1: 


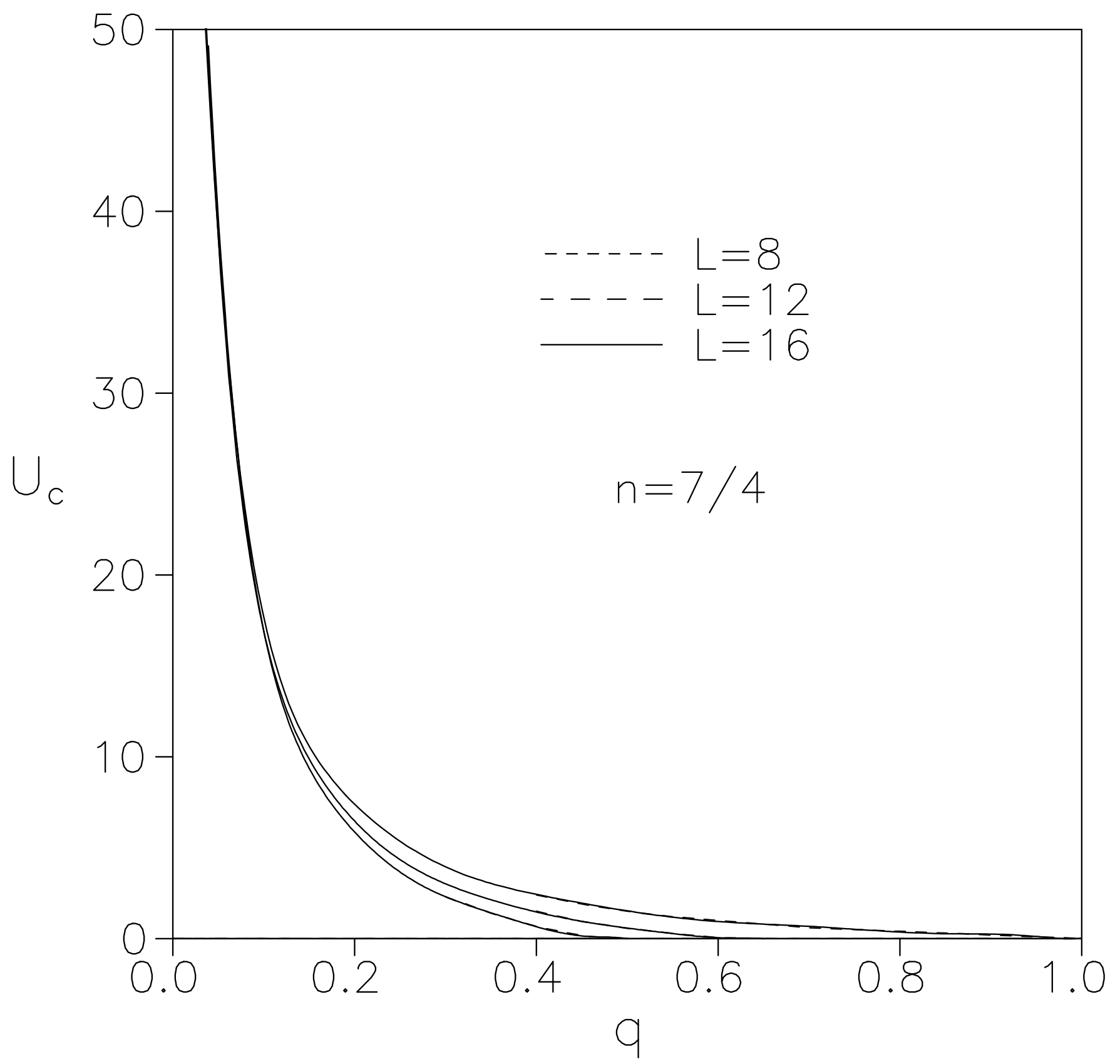

Figure 2: 